\title{
Anatomical features of Bougainvillea (Nyctaginaceae)
}

\author{
Sarah Chew* \\ *This study was conducted under the supervision of Professor John S. Greenwood, \\ Department of Molecular and Cellular Biology, College of Biological Science, \\ University of Guelph, Guelph, Ontario, Canada
}

\begin{abstract}
Two potted plants of Bougainvillea were obtained from a local nursery to explore their anatomical features using a variety of microscopical methods and stains. Various anatomical structures revealed were consistent with previous literature and these features were related to the natural habitat and established internal and external defenses of the plant. Results confirmed that the Bougainvillea is, in fact, a dicotyledonous plant that has evolved defensive features that permit its survival in hot and dry environments. Results indicated that features such as woody spines, the crystal inclusions in cells and the anomalous growth pattern are relatively unique to this species and important for its survival against predators.
\end{abstract}

$\mathrm{B}$ ougainvillea is a genus of bright flowering plants that belongs to the family, Nyctaginaceae [1]. This genus is native to Brazil and is named after Louis Antoine de Bougainville, the first man to discover and record its existence in 1786 [2]. Today, Bougainvillea is now a popular plant in Southern California, Florida, the Caribbean and other areas with tropical and warm climates [3]. It is best grown in direct sunlight with frequent fertilization and requires little water to grow [2]. Since the original discovery of this genus, many varieties have been identified and named. Of these varieties, Bougainvillea spectabilis wiild and Bougainvillea glabra appear to be more commonly studied and are cited extensively in the literature relative to others.

The primary use for Bougainvillea is as an ornamental because of its beautiful displays of colourful bracts. The specific cultivar that will be studied in this paper, "Barbara Karst" is known for its bright red bracts, but Bougainvillea cultivars have white, purple, lavender, and yellow bracts [1]. Bougainvillea can conform to a variety of habits such that, if left alone, it will assume the position of a sprawling shrub. If needed, this plant can be trained to cascade over walls, can be used as an espalier or can be transplanted into individual containers [1]. Bougainvillea tolerates trimming and shaping well, making it an easy plant to modify and control. Although Bougainvillea species respond well to horticultural use, this plant also has other useful properties. Recent research by Adebayo et al. has shown that extracts from Bougainvillea glabra leaves can have an anti-diabetic effect and can improve individual blood lipid profiles in rats [3].

Past anatomical research has emphasized the occurrence of anomalous secondary growth in stems and roots of Bougainvillea. Esau and Cheadle were two of the first researchers to reveal and explain anomalous secondary growth [4]. Following their work, researchers such as
Stevenson and Popham studied the ontogeny of the primary thickening meristem in seedlings [5]. Subsequently, Zamaski described vascular continuity in the primary and secondary stem tissues [6] and more recently, Carlquist reviewed the work of the authors listed above in revisiting secondary growth in plants such as Bougainvillea in addition to others [7].

In this paper, emphasis will be placed on exploring the anatomical features of Bougainvillea while considering previous literature and applying that knowledge to the cultivar, "Barbara Karst." In addition, information obtained about the environment in which Bougainvillea thrives will be used to try to understand why certain anatomical features exist in this genus and not others.

\section{MAterials AND Methods}

Two potted plants of Bougainvillea were obtained from a local nursery to explore their anatomical features using a variety of stains and materials and methods as outlined in Plant Anatomy Laboratory exercises by Peterson et al.[8].

Fresh samples of shoots, petioles, roots, leaves, bracts, spines, and flowers were sectioned transversely and longitudinally with a two-sided razor blade and stained using TBO to reveal various cell wall components [8]. Shoots and roots were sectioned transversely and stained using phloroglucinol to reveal lignified secondary walls, sudan III/IV to reveal structures containing lipid bodies, acid fuschin to reveal protein, and berberine to reveal suberin, lignin and callose. Berberine was particularly useful in identifying Casparian bands in the endodermis and exodermis of roots. Sections of roots stained with berberine were viewed under $\mathrm{UV}$ and blue light, using an epifluorescence microscope. $\mathrm{I}_{2} \mathrm{KI}$ was used to stain statoliths, distinctive structures found in the root cap. Other techniques 


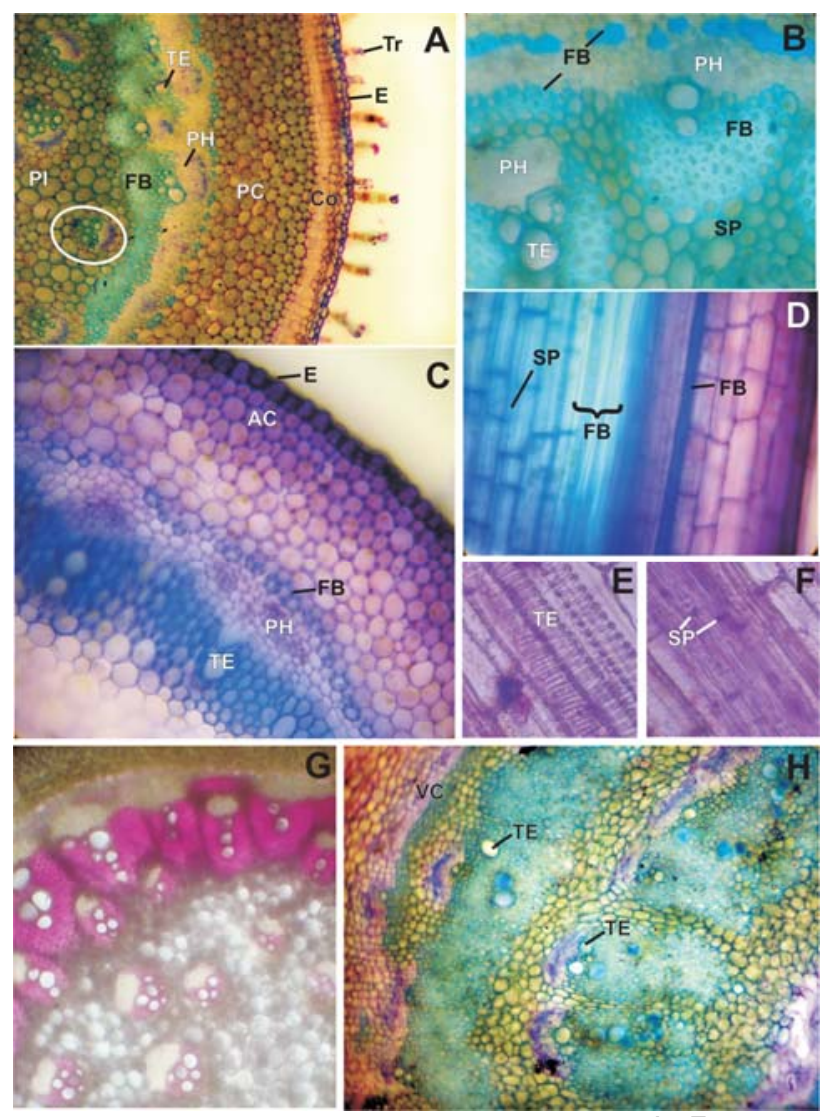

Figure 1. Anatomy of Bougainvillea stem. 1A. Transverse section of young stem stained with TBO, and revealing the surface trichomes ( $\mathrm{Tr}$ ), single cell layer thick epidermis (E), and underlying forming cork layer (Co) also known as the periderm, phloem (PH), tracheary elements of the xylem (TE), a ring of fibres (FB) and the pith (PI) containing small vascular bundles (oval). 1B. Magnified view of the vascular bundles revealing a thin layer of fibres (FB towards the periphery of the stem, with a thicker layer capping the phloem $(\mathrm{PH})$. Xylem tracheary elements (TE) are also surrounded by fibres and sclerified parenchyma (SP) 1C. Transverse section of a very young stem stained with TBO. A layer of angular collenechyma (AC) having irregularly thickened primary cell walls lies immediately under the epidermis (E) . FB, fibres; $\mathrm{PH}$, phoem; TE, tracheary elements of the xylem 1D. Longitudinal section of young stem stained with TBO illustrating fibres (FB) and sclerified parenchyma (SP). $1 E$ and F. Longitudinal section of stem stained with acid fuschin revealing the helical and scalariform nature of the cell wall thickenings of xylem tracheary elements (TE in $1 \mathrm{E}$ ) and sieve plates in the sieve tube members of the phloem (SP in 1F) 1G. Transverse section of a young stem stained with phloroglucinol $\mathrm{HCl}$. Pink staining reveals lignified cell walls of the xylem tracheary elements and fibres. $1 \mathrm{H}$. Transverse section of older stem revealing anomalous secondary growth. Successive vascular regions are added as the stem expands laterally. Two distinct regions of mature vasculature are shown with a tracheary element (TE) of the xylem indicated in each. A new layer of vascular cambium (VC), which will give rise to new xylem and phloem, has been initiated in the parenchyma more proximal to the outside of the stem .

used that were particular to the shoot included aniline blue to reveal callose, a cell wall component of phloem.

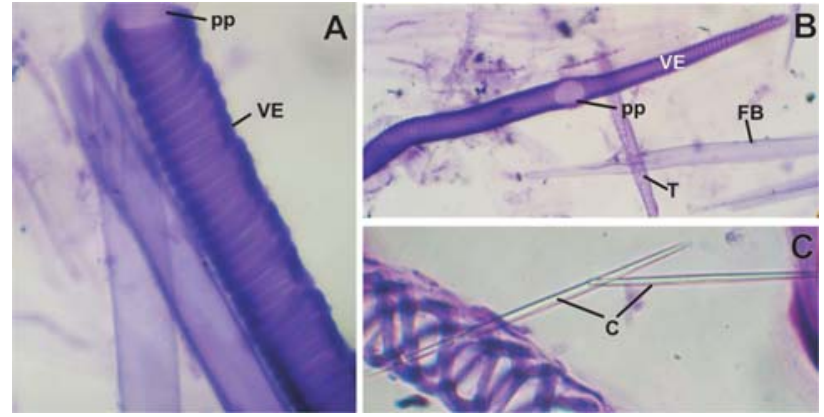

Figure 2. Stem macerates allow identification of elements of the vasculature and raphide crystal. All images stained with TBO. 2A. A vessel element (VE) with simple perforation plate (pp) 2B. Elongated vessel element (VE) with simple perforation plate (pp) lies over a tracheid $(\mathrm{T})$ having bordered pits. $\mathrm{FB}$, fibre $2 \mathrm{C}$. Numerous spear-shaped raphide crystals ( $\mathrm{C}$ ) were found in all macerates.

In addition to these techniques, macerates of shoots and petioles were obtained following the method outlined in Peterson et al., 2008 [8] and stained using TBO to reveal tracheids, fibres and vessel elements.

Longitudinal sections of the spine and the floral apical meristem were embedded in paraffin wax and sectioned using a microtome according to the techniques described in Peterson et al. [8]. Sections were mounted on a slide, stained with TBO and viewed using a compound microscope to reveal crystal idioblasts, leaf primordia and trichomes [8]. Techniques unique to the leaf and bract included clearing, using $10 \%$ aqueous $\mathrm{KOH}$ at $55 \mathrm{C}$ for the leaf and boiling $95 \%$ ethanol for the bract, to reveal venation patterns, and to locate the midvein, lateral veins, vein endings, areoles and trichomes [8]. Both cleared leaves and bracts were stained using TBO to reveal mid-vein, midrib parenchyma, xylem and phloem, palisade and spongy mesophyll, stomata, guard cells, glandular trichomes and crystals. Unique to the leaf, nail polish impressions were made of the adaxial and abaxial epidermal surfaces to reveal stoma, guard cells, subsidiary and pavement cells.

\section{RESULTS}

\section{Stem}

Transverse and longitudinal sections were made of the young stem and stained to help reveal the characteristics of the Bougainvillea anatomy. Starting at the periphery of the stem and progressing inward, numerous multicellular trichomes arise from a single layer of epidermal cells. Just inside the epidermis, there are successive layers of newly arising cork cambium, parenchyma cells, phloem, tracheary elements of the xylem, and a thick band of fibres, all surrounding the central pith. Embedded in the pith are vascular bundles which are themselves composed of xylem and phloem (Figure 1A). A closer examination of individual vascular bundles reveals fibres, both removed from and immediately surrounding the vasculature, the latter being composed of both phloem and tracheary elements of the xylem, 


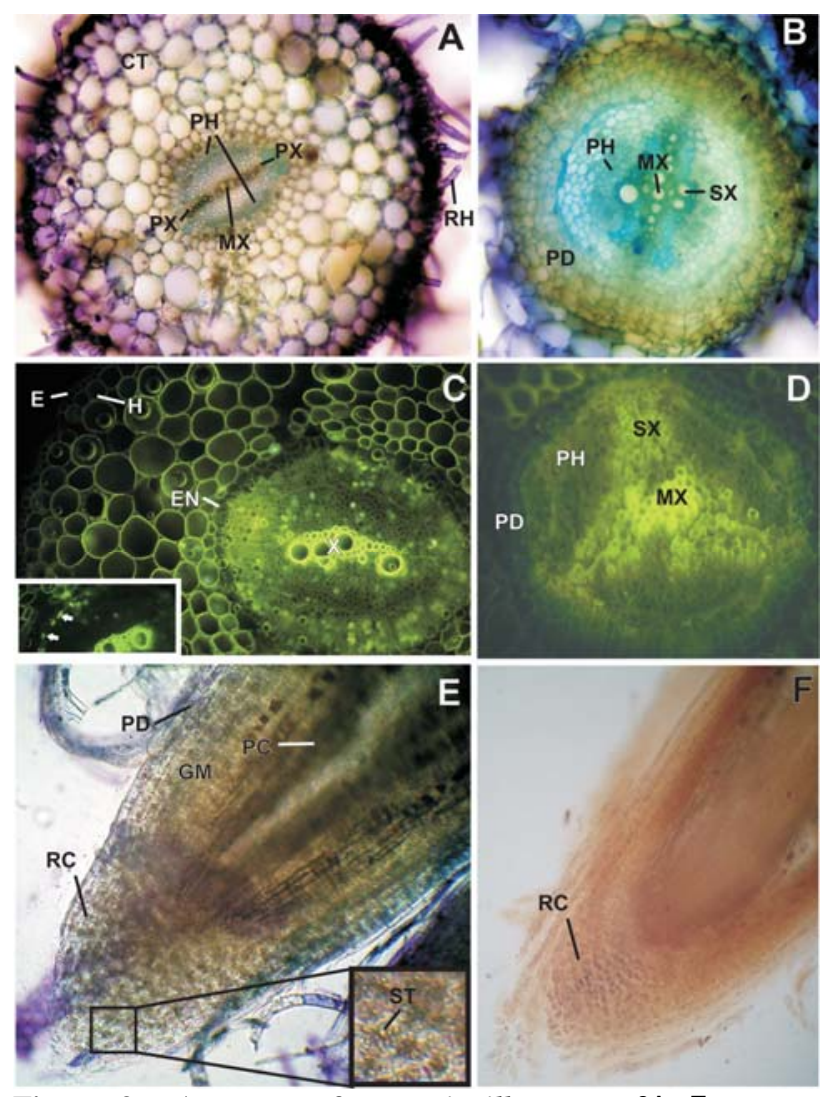

Figure 3. Anatomy of Bougainvillea root. 3A. Transverse section of a very young root stained with TBO, revealing the diarch nature of the vasculature. The xylem consists of two protxylem (PX) poles with metaxylem (MX) between. Archs of phloem (PH) lie outside the xylem. Cortex (CT) surrounds the vasculature, which in turn is surrounded by an epidermal layer having numerous root hairs (RH) 3B. Transverse section of older root stained with TBO Secondary growth has been initiated as indicated by the addition of secondary xylem adjacent to the metaxylem, phloem $(\mathrm{PH})$ is more developed, and the periderm (PD) is starting to form with the lateral expansion of the root. 3C. Transverse section of young root stained with berberine hemisulfate and viewed under UV light shows the epidermis $(E)$, hypodermis $(H)$, xylem $(X)$ and the region of the endodermis (EN). Viewing under blue light confirms the region of the endodermis by reveling the Casprian band $\mathrm{s}$ associated with the cells of this layer (inset, arrows). 3D. Transverse section of old root stained with berberine hemisulfate and viewed under blue light illustrates the expansion of the secondary xylem (SX) around the region of meatxylem (MX). The blue tinge reveals the initiation of a periderm $(P D)$, similar to that seen in $3 B$, with suberin in these cells being responsible for the blue fluorescence. 3F. Longitudinal section of root tip stained with TBO and illustrating the locations of the procambium $(\mathrm{PC})$, ground meristem (GM) and protoderm (PD). The apical meristem of the root is protected by a root cap (RC) which has statoliths (inset, ST). $3 \mathrm{H}$. Longitudinal section of root stained with $\mathrm{I}_{2} \mathrm{KI}$ revealing an open organization of the root apex and demonstrating that the statoliths are most likely starch (purple-black deposits in root cap).

presumably vessel elements. Interestingly, parenchyma lying near the fibres has thickened, sclerified cell walls (Figure 1B). In very young stems (Figure1C), angular collenchyma, having primary cell walls thickened in the corners, lies immediately below the epidermis. Longitudinal sections of stem are shown in Figure 1 D, E, F. Fibres and sclerified parenchyma are illustrated in figure 1D. Figure 1E reveals the helical and scaliform secondary cell wall thickenings of the trachaery elements of the xylem. Peripheral to the xylem, phloem sieve tube members are identified by the presence of sieve plates (Figure 1F). Phloroglucinol staining of a transverse section of a young stem reveals the highly lignified nature of the fibres associated with the xylem (pink staining Figure 1G). The anamolous secondary growth seen in Bougainvillea is illustrated in a transverse section of a more mature stem (Figure 1H), where individual layers of vasculature have been deposited. A new vascular cambium has been initiated in the parenchyma layer toward the outside of the stem, the successive vascular cambia defining anomalous growth.

Macerates of the stem stained with TBO similarly revealed the helical secondary wall thickenings in some of the vessel elements (Figure 2A). Fibres, vessel elements with simple perforation plates, and tracheids with bordered pits (Figure 2B) and individual raphide crystals (Figure2C) were also seen.

\section{Roots}

Young roots in primary growth sectioned transversely and stained with TBO, revealed the formation of a diarch vaxcular arrangement with xylem having two protoxlyem poles with metaxylem between. The developing phloem formed two arches surrounding the xylem. Young roots also showed an extensive number of root hairs (Figure 3A). As secondary growth began in the older roots (Figure 1B), secondary tracheary elements were added outwards from the metaxylem and phloem began to surround the secondary xylem. The formation of a periderm was initiated at this point.

Roots that were stained with berberine and viewed under UV light confirmed the same results as the TBO, showing the diarch arrangement, cortical parenchyma, hypodermis and epidermis (Figure 3C). The berberine stain viewed under blue light revealed the diarch arrangement with Casparian bands formed in the surrounding endodermis (Inset Figure 3C). It should be noted that a triarch arrangement was also identified in older regions of the root and when stained with berberine and viewed under blue light, tissue having a blue fluorescence, owing to the presence of suberin, was revealed (Figure 3D).

Longitudinal sections of the root tip stained with TBO revealed root cap cells, protoderm, procambium and ground meristem (Figure 3E). A closer view of these root cap cells showed that statoliths are present (Inset Figure 3E). Longitudinal sections stained with $\mathrm{I}_{2} \mathrm{KI}$ revealed an open organization of the root system and confirmed that statoliths contain starch (purple/black staining in root cap) (Figure 3F). 


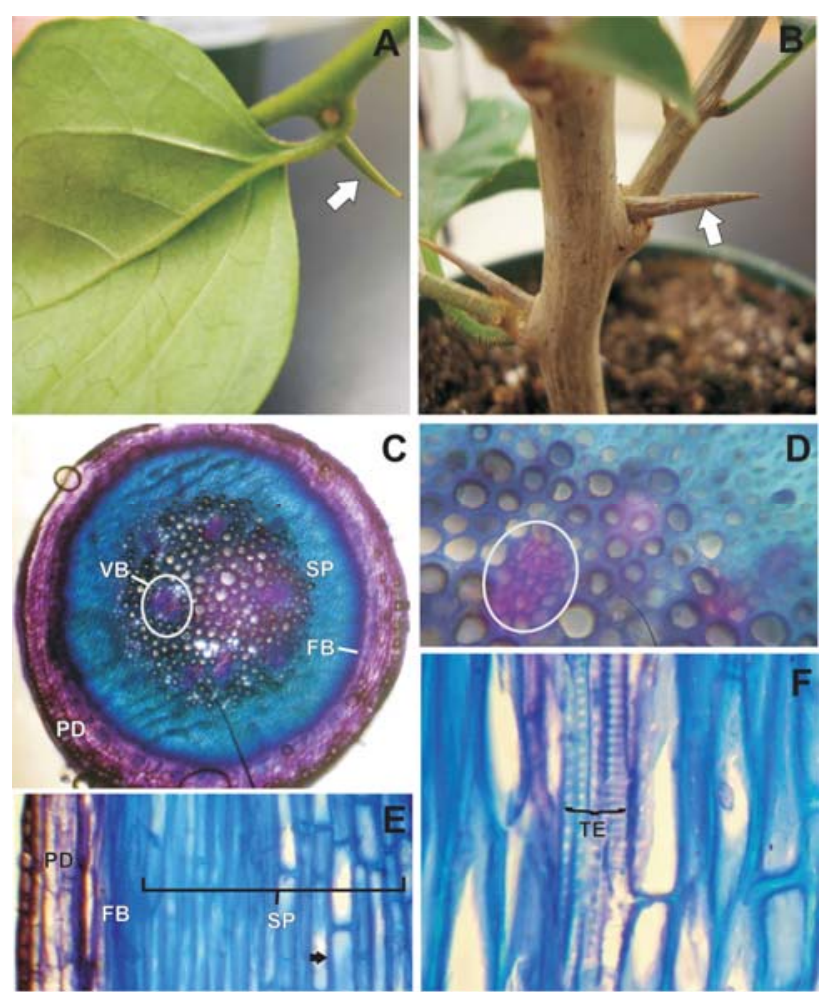

Figure 4. Anatomy of Bougainvillea protective spines. $4 \mathrm{~A}$ and B. Spines arise as modified leaves (arrow, 4 A) and become wood y with age (4 B). 4C and D. Transverse section of spine stained with TBO revealing a well developed periderm (PD) surrounding a thick sclerenchymatous layer composed of fibres (FB) and sclerified parenchyma (SP). Small vascular bundles (VB) are present in a central pith-like region $(4 \mathrm{C})$. The vascular bundles contain relatively few cells (oval, $4 \mathrm{D}$ ). 4E. Longitudinal section of spine stained with TBO and focusing on the sclerenchymatous layer reveals long fibres (FB) beside a region of short rectangular sclerified parenchyma cells (SC) having thickened cell walls. 4F. Longitudinal section of spine stained with TBO and focusing on the small vascular bundles. Xylem tracheary elements individually have bordered pits (presumably tracheids), and helical wall thickenings (tentatively vessel elements).

\section{Spines}

Spines of the Bougainvillea plant are very solid structures, which appear green when young (Figure 4A) and woody (Figure 4B) when mature. Spines sectioned transversely and stained with TBO revealed a very thick periderm, followed by a ring of fibres, overlaying a ring of sclerified parenchyma. Within this ring, a parenchymatous pith contains some vascular bundles (Figure 4C), albeit very small (Figure 4D). Longitudinal sections reflect what is seen in the transverse section, showing fibres and extensive sclerified parenchyma. Note that the sclerified parenchyma have very thickened cell walls (Figure 4E). A closer view of this longitudinal section reveal some tracheary elements composed of vessel elements and tracheids seen among the fibres, albeit small and difficult to find (Figure4F).

\section{Leaves}

Leaves of the Bougainvillea plant display an alternate, simple arrangement along the stem with an undulate leaf margin and ovate shape (Figure 5A). Leaves and bracts that were sectioned transversely and stained with TBO revealed a variety of structures including the midvein (xylem and phloem), midrib parenchyma, palisade and spongy mesophyll, covering trichomes and raphide crystal bundles (Figure 5B). A closer view of the transverse leaf viewed using brightfield and cross polarizing microscopy confirmed that these structures contained crystals (Inset Figure 5B). Intact leaves viewed under the dissecting microscope revealed the venation pattern of the leaf, showing the midvein, lateral veins, areoles and trichomes (Figure 5C). A closer view of a cleared leaf stained with TBO revealed numerous multicellular trichomes on the adaxial surface, although trichomes have been found on both surfaces (Figure5D). Epidermal peels revealed that stomata complexes are generally found on the abaxial side of the leaf (Figure 5E) while nail polish impressions further demonstrated this finding (Figure 5F). Nail polish impressions also revealed that stomata were absent on the adaxial surface and that there were differences in the shapes of the pavement cells found on the abaxial versus the adaxial side of the leaf (Figure 5G).

\section{Flower}

Longitudinal sections of the flower left unstained revealed the internal anatomy including the perianth, filaments, anthers and pollen grains (Figure 6A). On the external surface of the perianth, pink-purple rrichomes were evident. A macro photo shows the tubular shape of the flower, surrounded by the bright pink bract (Inset Fig 6A). Cross polarization of the flower also revealed these pink-purple trichomes as well as pollen grains and numerous crystal bundles seen throughout the entire organ (Figure 6B).

\section{DISCUSSION}

Anomalous growth has been characterized by the presence of multiple cambia that are ontogenetically related yet forming independently of one another [4]. Each year, parenchyma cells in the stem will dedifferentiate and become meristematic again forming new phloem and xylem tissue where phloem is always to the periphery and xylem to the middle of the stem [4]. As growth continues, the vasculature appear to be randomly dispersed due to the activity of each independent cambium. The organization of the vascular bundles themselves appear to exhibit a collateral arrangement with fibres, sclerenchmya and possible angular collenchyma found surrounding these bundles. These cell types provide extra protection and add support to the growing stem. The Bougainvillea plant is also characterized by its woody stem as it matures and is accompanied by a periderm layer. Sections of the mature periderm were difficult to obtain. The periderm is normally present in older 


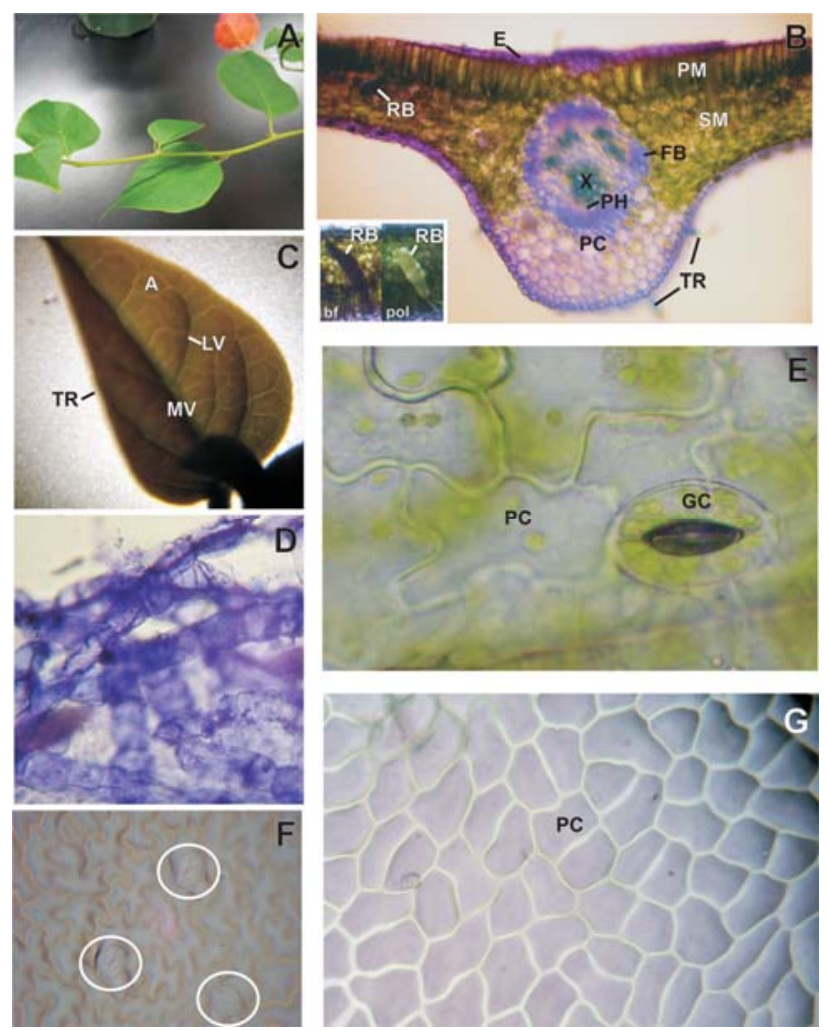

Figure 5. Morphology and anatomy of Bougainvillea leaves. $5 \mathrm{~A}$. Leaves of Bougainvillea are arranged in a simple alternate arrangement on the stem. Leaves have ovate shape with undulate margin. 5B. Transverse section of leaf through the midvein and stained with TBO reveals a fairly typical arrangement of photosynthetic mesophyll, with a single layer of palisade mesophyll (PM) nearest the adaxial (upper) surface and spongy mesophyll (SM) below. The epidermis $(E)$ is a single cell layer with multicellular trichomes (TR, difficult to distinguish in this section) The vascular bundle of the midvein has regions of xylem $(X)$ and phloem $(\mathrm{PH})$ surrounded by scattered regions of fibers (FB). There is an extensive layer of parenchyma tissue (PC) between the lower epidermis and the vascular bundle, which may aid in support. Bundles of raphide crystals (RB) are scattered throughout the mesophyll, the inset showing that the dense structures seen in brightfield microscopy (bf) contain crystal structures as observed under cross polarized light (pol). 5C. Intact leaf viewed under dissecting microscope shows net-venation pattern of the Bougainvillea leaf, with mid-vein (MV), lateral veins (LV) and areoles (A). Trichomes are numerous on the abaxial surface. 5D. Close up view of cleared leaf stained with TBO shows the very numerous multicellular trichomes of the leaf, the only structures seen in this image. 5E. Close up of epidermal peel of the abaxial surface of the leaf illustrating the guard cells (GC) of the stomtal complex and pavement cells (PC) of the epidermis. The stomatal complex lacked obvious subsidiary cells. 5F and G.. Nail polish impressions of the abaxial and adaxial leaf surfaces illustrate that stomatal complexes exist only on the abaxial surface (ovals, 5F). Pavement cells of the abaxial surface have the shape of jigsaw puzzle pieces, whereas those on the adaxial surface $(\mathrm{PC})$ are more regular in shape.

Bougainvillea stems and adds extra protection to the stem as it grows. The suberized phellum would be of great benefit to the Bougainvillea plant growing in the hot, dry climate as it helps to seal off water loss. Very young shoots did not have mature periderm but rather an epidermal layer characterized by primary cell walls. Just inside this epidermis, angular collenchyma (characteristic of stems) are found which aid in the support of the rapidly elongating stem and then parenchyma containing chloroplasts, signifying the photosynthetic activity of the young stem. The thin layer of fibres found just outside the vascular bundles exists to protect the outer most layer of phloem. Moving further inwards, a second fibre band with sclerified parenchyma appears. This thick fibre band surrounds the innermost vascular bundles. As secondary growth proceeds, some of the parenchyma cells found within and outside of this band will become meristematic and contribute to the formation of new vascular cambia and a new layer of fibres, providing them with extra strength and flexibility as the stem continues to grow expand.

The diarch and triarch arrangements found within the root are further evidence that Bougainvillea is a dicotyledonous plant. Esau \& Cheadle [4] and Stevenson \& Popham [5] also took note of these two arrangements and in particular, the diarch arrangement in young roots of Bougainvillea. The switch to a triarch arrangement occurred due to the widening diameter of the older root and the addition of secondary xylem and phloem. In the older sections of the root, much of the cortex had been crushed and sloughed off, including the endodermis and the pericycle and leaving the xylem and phloem surrounded by a newly forming layer of periderm, ultimately to protect this area of the root from losing water to the environment. According to Esau and Cheadle, much of the phloem associated with the normal secondary xylem increment is crushed during the expansion growth of the root, thus only the most recently formed phloem is active [4].

Sections stained with berberine and viewed under blue light did reveal Casparian bands. Due to the dry climate in which Bougainvillea thrives, it would have adapted these to help keep water locked in its roots [8]. Another adaptation that also would have helped with water retention is the cortex which appears to contain secondary wall deposits in its cell walls. TBO and $\mathrm{I}_{2} \mathrm{KI}$ revealed statoliths consisting of starch in the root cap cells; these aid in directing the downwards growth of the root, a process known as gravitropism [8].

The sharp structures located near leaf buds on the stem are referred to as spines and are modified leaves. These structures are another feature of this plant's defence system. Young spines are green and involved in photosynthesis. As these structures age and become woodier, their photosynthetic abilities cease. The vascular bundles also become much smaller and eventually collapse. This allows for the plant to divert its energy sources to other structures. Eventually, a mature spine will be mostly composed of sclerified parenchyma, fibres, and a thick periderm which aid in creating a strong structure for protection and deterrence to pests. 


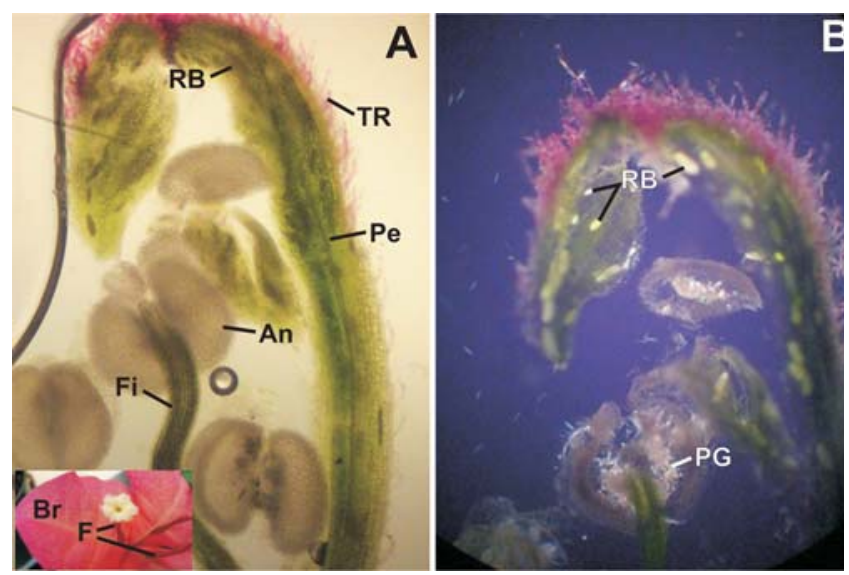

Figure 6. Mophology and anatomy of Bougainvillea flowers. 6A and B. Bougainvillea flowers are perfect flowers $(F)$ that reside in groups of three above colourful leaf-like bracts (Br, inset $6 \mathrm{~A})$. The fused perianth is slender and tubular in nature (inset $6 \mathrm{~A}, \mathrm{PE} 6 \mathrm{~A}$ ) and has numerous trichomes (TR) on the outer surface (6A). Stamens with anthers (AN) and filaments (Fi) were obvious within the sectioned flower. As with the leaves, bundles of raphide crystals are present in the perianth, presumably as a protective measure against herbivores. $6 \mathrm{~A}$, bright field unstained, $6 \mathrm{~B}$ cross polarizing microscopy. Pollen grains (PG), having thick almost crystalline cell walls are revealed using polarized light (6B).

The leaves of the Bougainvillea plant have adapted to a hot, dry climate in several ways. Their flat surface makes it ideal for maximum light interception while the wide surface allows for a denser distribution of stomata complexes. The leaves also contain a thick epidermal layer, covered by a thin cuticle layer (cuticle layer not shown). The cuticle layer allows the leaf to prevent water loss in the hot environment [10]. The stomata complexes, all found on the abaxial side of the leaf, allows the leaf to carry out gas exchange during the hot day without the direct heat of the sun drying out the leaf. Other adaptations of the Bougainvillea leaves include a dense covering of epidermal hairs and the distribution of raphide crystals. The epidermal hairs, also known as covering trichomes, function to deter herbivory by insects, prevent organisms from inhabiting the surface, screen out some UV light and reduce water loss by forming a buffer against air flow [11]. The raphide crystals are a part of the plant's passive defence system and act in deterring herbivory. These crystals have also been found throughout many other structures including the stem, root, flower and spine.

The Bougainvillea flower is a true, perfect flower that is small and tubular in shape. The actual flower itself is white or yellow and is surrounded by showy, vibrant bracts [9]. The colourful bracts are, in fact, not petals but modified leaves, adapted to attract pollinators to the colourless and scentless flowers residing on the upper surface. The research of $\mathrm{Xu}$ et al. aided in determining structures found in the Bougainvillea flower such as the perianth, filaments, anthers and pollen grains [12]. These researchers also made note of the pink-purple trichomes protruding from the tubular perianth. According to their research, these hairs are referred to as a stylar brush, which might be responsible for inhibiting contact between the flower's pollen mass and stigma between the cluster of flowers found on each bract, reducing sterility in this plant. They have also proposed that the stylar brush contains betalains, (a compound responsible for the purple colour found in beets), which accumulate in the bracts and perianth as they grow. It has been strongly suggested that these betalains function to protect the plant from the stresses of high temperature and strong light which coincides with the hot and dry climate Bougainvillea thrives in [12]. As noted in the leaf, the tubule flower and bracts also contain raphide crystals which again serve as a passive defence mechanism for this plant.

Most of the features of the anatomy of Bougainvillea are fairly typical of plants that exist in temperate to tropical environs. Features that are unusual in Bougainvillea include the spines, the crystal inclusions in cells and the anomalous secondary growth pattern. These adaptations may be more related to defence against herbivory. The spines provide the plant with an obvious defence against large herbivores. The spear-shaped raphide crystals, most likely composed of calcium oxalate, would act as both a physical and a toxic chemical deterrent against both small and large herbivores [4]. Finally, the anomalous secondary growth with the accompanying layers of fibres that are laid down result in scale-like pattern of fibrous protection around the vasculature. This would be difficult for any herbivore to penetrate. Thus, in attempting to relate plant anatomy to environment, we must consider both the abiotic and biotic factors in that environment.

\section{REFERENCES}

1. Gilman, F., E. 1999. Fact sheet: Bougainvillea spp. Available from the Environmental Horticulture Department, Florida U.S. Publishing.

2. Hammad, I. 2009. Genetic variation among Bougainvillea glabra cultivars (Nyctaginaceae) detected by Rapd markers and Isozymes patterns. Journal of Agriculture and Biological sciences. 5 (1): 63-71.

3. Adebayo, I.G., Alabi T. O., Owoyele, V., B., and Soladoye, O., A. 2009. Anti-diabetic properties of the aqueous leaf extract of Bougainvillea glabra on alloxaninduced diabetic rats. Rec. Nat. Prod. 3 (4): 187-192.

4. Esau, K., and Cheadle, I,V. 1969. Secondary growth in Bougainvillea. Ann. Bot. 33: 807-819.

5. Stevenson,W.D., and Popham, A.R. 1973. Ontogeny of the primary thickening meristem in seedlings of Bougainvillea spectabilis. Am. J. Botany. 60 (1):1-9.

6. Zamski, E. 1979. Vascular continuity in the primary and secondary stem tissues of Bougainvillea (Nyctaginaceae). Ann. Bot. 45: 561-567

7. Carlquist, S. 2007. Sucessive cambia revisited: ontogeny, histology, diversity and functional significance. Torrey J. Bot. 134 (2): 301-322. 
8. Peterson L., Greenwood J., Lee B., McClellan D. 2009. Plant Anatomy (Bot*3410) Laboratory Exercises. Department of molecular and cellular biology. University of Guelph

9. Kobayashi, D.K., McConnell, J., Griffis, J. 2007. Bougainvillea. Available from the Department of Tropical Plant and Soil Sciences, published by the College of Tropical Agriculture and Human Resources
10. Purves, K.W., Sadava, D. Orians, H.G., Heller, C.H. 2004. The plant body. In The science of biology. Sinauer Associates, Inc., Sunderland, MA. Pp.

11. Peterson, R. L., Peterson, A.C, Melville, H.L. 2008. Teaching Plant Anatomy. NRC Press, Ottawa, Ontario.

12. Xu, S., Huang, Q., Shu, Q., Chen, C., Vick, A.B. 2009. Reproductive organography of Bougainvillea spectabilis Willd. Scientia Horticulturae. 120 (3): 399-405. 